\title{
Exploring Mentoring in the Context of Team Science
}

\author{
Linda S. Behar-Horenstein and Alena Prikhidko
}

\begin{abstract}
Despite an increasing amount of research into team science and collaboration, little is known about post-doctoral scholars' pathways to becoming independent researchers, particularly as it relates to the mentoring relationship. The purpose of our study was to explore if and how the team science framework promotes collaboration and the development of independent researchers from mentees' and mentors' perspectives. A convenience sample of faculty mentors and postdoctoral mentees, participated in semi-structured interviews. The analysis revealed three themes for the mentees: working with others, evaluating mentoring, exploring resources, and five themes for the mentors: elaborating on scholarly productivity, mentoring style, setting team culture, strategies to develop T32 scholars, and evaluating program qualities. The findings showed that scholars were engaged in an atmosphere of collaboration fostered by their mentors and that mentors encouraged scholars' capacity for scientific thinking. Future studies should acquire the perspectives of all team members to examine how teams function.
\end{abstract}

\section{Keywords}

clinical translational research; mentor-mentee relationships; mentoring; qualitative research; postdoctoral education; team science

\section{Introduction}

Since the enactment of legislation in 1974, the National Research Service Awards NRSA program, also referred to as the T32 program, has been the primary means of supporting predoctoral and post-doctoral research training programs. These programs aim to develop and or enhance research training opportunities for individuals interested in biomedical, behavioral and clinical research careers that are relevant to the National Institutes of Health (Ruth L. Kirschstein Institutional National Research Service Award. NRSA). Mentoring the next generation of independent research clinician scientists is fundamental to the success of these awards. At the health science center institution where our study was conducted, a dedicated focus on promoting team science and moving away from reliance on the model of only one mentor has been ongoing. The researchers were interested in developing a better understanding of the ways in which the mentor-mentee relationships in the T32 program facilitated or hindered effective team-based research and practice, and in identifying how

CORRESPONDING AUTHOR: Linda S. Behar-Horenstein, Ph.D., Distinguished Teaching Scholar and Professor, Colleges of Dentistry, Education, \& Pharmacy, University of Florida, Gainesville, FL 32610-0208, Tel: 352682 0768, Lsbhoren @ ufl.edu,. Alena Prikhidko, M.S. Doctoral Candidate, Counselor Education, College of Education, University of Florida, Gainesville, FL 32610-0208, Tel: 352757 6129, a1978@ufl.edu. 
those relationships impacted knowledge integration and productivity (Stokols, 2013). Despite the increasing amount of research into team science and collaboration, little is known about postdoctoral scholars' pathways to becoming independent researchers and how mentoring influences those trajectories. In our study, warranted by the National Science Foundation and the National Cancer Institute, the researchers sought to clarify the outcomes related to its investments in large science groups (e.g., research centers) and to augment their understanding of how best to support and manage team science (Croyle, 2008; 2012)

\section{Team Science and Mentoring}

In the last few decades, researchers in almost all branches of science have turned towards teams, also known as team science, as a unit of scientific knowledge production. Team science is a collaborative approach to addressing scientific challenges that utilizes the strengths and expertise of professionals trained in different fields. Team science may use coordinated teams of investigators with diverse skills and knowledge to study and resolve scientific problems that have multiple causes or are a byproduct of complex social problems (National Cancer Institute, Cite Team Science Toolkit retrieved from https:// www.teamsciencetoolkit.cancer.gov/public/WhatisTS.aspx February 5, 2017). Teams are not only used in science. They are also used as an approach to instruction as well. In our study, the reference to team science focuses solely on knowledge integration of interdisciplinary or transdisciplinary projects. However, not all research groups or teams that function within the team science framework are the same. Research teams may be comprised of investigators from the same or different fields, and vary by size, organizational complexity, and at times geography, when departments at several universities collaborate on a common research agenda (Bennett \& Gadlin, 2012). Research teams may include as few as two individuals engaged in collaboration to many individuals. These teams are often led by faculty researchers that employ students who are interested in their research. These students can range from undergraduate, graduate, to postdoctoral fellows. Also, consistent with the team science approach is that doctoral students and postdoctoral fellows typically have comentors. The primary mentor is usually a basic scientist or a clinician. If the primary mentor is a basic scientist, then the co-mentor is a clinician and vice versa. Thus, their committees are often of primary and secondary mentors (co-mentors), and someone with a particular methodological expertise, similar to the composition of a doctoral committee. The participants and subjects of this inquiry were postdoctoral fellows, also referred to as T32 scholars, who were funded by a National Research Service Awards (NRSA) postdoctoral training program (T32), designed to ensure that a diverse and highly trained workforce is available to meet the needs of the nation's biomedical, behavioral, and clinical research agenda,

To be considered for a T32 fellowship, potential postdoctoral fellows contacted a faculty member who had been awarded an NRSA and applied for a funded position. Consistent with the concept of team science, T32 scholars are likely to discuss and seek advice beyond their primary mentor from a secondary mentor or other faculty whose expertise brings new insight to their research study. T32 scholars also typically attend seminars, take additional courses, participate in their primary or secondary mentor's journal clubs or group meetings. Thet 
receive guidance and direction from a committee who advises them as they progress towards research and professional goals.

Team science researchers now typically outnumber solo scientists in the production of highimpact science (Börner et al., 2010; Falk-Krzesinski et al., 2011). A team science program may occur with cross-disciplinary, multidisciplinary and interdisciplinary sub-specialty researchers. Fiore (2016) described interdisciplinary research performed in a team as team science, in which collaboration leads to the design of "new types of complex empirical approaches along with integrated analysis combining methods and concepts from participating disciplines [emphasis present]" (p. 254). Team science and mentoring are intertwined; to perform well in a team, arguably one needs to have had a good mentor at some time (Guise et al., 2017). Mentors play a central role in guiding the mentee's (the postdoctoral/ T32 scholar) development as an emergent independent scientist by: (a) being a sounding board, (b) discussing formative failures and successes; (c) articulating research goals, (d) selecting prospective employment opportunities, and (e) serving as a helpful adversary who challenges the scholar's scientific reasoning and ensures that the T32 scholar can defend his science.

Good or effective mentors have been described in various ways. However, there seems to be an agreement that good mentors provide career guidance, offer emotional support and focus on work/life balance (Straus, Johnson, Marquezm \& Feldman, 2013). Characteristics of effective mentors include: altruism, honesty/trustworthiness, active listening, mentoring and professional experience and networks, accessibility, and ability to identify mentees' strengths and weaknesses. Good mentors ensure that teams work collaboratively and effectively to facilitate the attainment of the researchers' goals. These concepts -- team science and evaluating mentors are described in the next sections.

\section{Science of Team Science}

Researchers identified a new field of inquiry named "the 'science of team science' (SciTS pronounced 'sahyts')" (Börner et al., 2010, p. 1) in which they strive to understand how science teams are organized, the mechanisms of communications and how research is conducted in those teams (Fiore, 2008). As Stokols (2013) pointed out, the purpose of studying SciTS is "to better understand the circumstances that facilitate or hinder effective team-based research and practice and to identify the unique outcomes of these approaches" [as it relates to] productivity, innovation, and translation" (p. 4).

Börner et al. (2010) proposed a multi-level mixed-methods approach to explore or investigate SciTS, suggesting studies at macro-, meso- and micro-levels. The macro-level examines team functioning in the population and investigates the patterns of collaboration, which influence the growth of knowledge. The meso-level or group level studies, looks at interaction patterns or the amount and nature of intra-team communications. The micro-level is related to the individual within a team and pertains to intrapersonal characteristics relevant for the success of a team and educational approaches related to training team scientists. Lotrecchiano (2013) found four dominant social mechanisms in the analysis of interactions at the micro/meso level (a) change, (b) kinship, (c) tension, and (d) heritage. He determined 
that these components, key features of team science, played an integral role in informing the integration and engagement within teams.

Educating future team scientists is one of the many challenges in the SciTS. Other problems for consideration relate to maintaining intellectual property rights among researchers and universities, managing promotion, and searching for the factors of team success. Somewhat similar to Börner et al. (2010), Fiore (2016) proposed systematic training needs analysis at three levels (a) organizational - to identify the need for training and the degree of its support from the institution, (b) task - to identify the content, methodology or type of data analysis needed for a team's success, and (c) team - to define the needs for training within a research team.

As Croker, Trede and Higgs (2012) pointed out, "despite the increasing amount of research in teams and collaboration, achieving sufficient depth and breadth of understanding to inform the development of complex collaborative practice remains a challenge" (p. 13). They conducted semi-structured interviews to explore the experiences of collaborating in nine rehabilitation health-care service teams. Eight interdependent dimensions central to the collaborating experiences emerged. Five named interpersonal dimensions of endeavor included (a) engaging positively with diversity, (b) entering into the form and feel of the team, (c) establishing ways of communicating and working together, (d) envisioning frameworks for a patient's rehabilitation, and (e) effecting changes in people and situations. Three other dimensions, called reviewing, operated across the five endeavor dimensions and included (a) reflexivity, (b) reciprocity, and (c) responsiveness. Wildman and Bedwell (2013) suggested "that educators teaching courses on teams may want to practice what we preach (p. 381). Thus, they recommended the mentors clearly describe for students, what team science is and provide them with practice and hands-on experiences while working on teams. They asserted that demonstration, practice, and feedback were primary links to science and practice, while demonstration was considered fundamental to the translation of knowledge into the practice.

Since the early 1990's, researchers have believed that the success of transdisciplinary team science is generated though multilevel interventions (Bennett, \& Gadlin, 2012; Hall et al., 2008). However, little is known about diverse readiness factors, which impact the effectiveness of the team science. Hall et al., suggested looking at readiness from a socioecological perspective and developing a readiness framework by exploring the team's readiness at various levels. For example, they found that team cohesiveness and communication skills improved at the interpersonal level with workshop modules that aimed to foster teams' success. Kauffelld and Lehmann-Willenbrock (2011) observed that problem-solving interactions and action planning were correlated with the satisfaction of team science meetings and that better meetings were associated with increased ratings of success among teams. Team success also been found to be dependent upon training in transdisciplinary science. Thus, teams should incorporate the "broader models of transdisciplinary training that encompass the needs of all stakeholders including senior investigators, junior investigators, post-doctoral scholars, graduate students, and research support staff (...) into the overall infrastructure of team science" (Hall et al., 2008, p. S246). 
Translating knowledge into practice cannot be done without mentoring -- it is pivotal to the learning process. Mentoring, regarded as vital in the development of scientists, has been found to support the professional development of a junior faculty and to promote the retention of clinician-scientists (Byington, et al., 2016). Despite its importance, there is no single way to describe the mentoring process and the role of a mentor in relation to the translational outcomes. However, the manner in which mentoring is evaluated is crucial to understanding what mentoring in practice looks like.

\section{Evaluation of Mentoring}

As a term, mentor is widely used in academic environments, but there is no consensus on its definition. Meagher et al. (2011) agreed that lacking was widespread agreement on the competencies thought to characterize effective mentoring experiences. The researchers provided an evaluation of research methods and those measures used to "assess the mentormentee relationship, mentor skills and mentee outcomes" (p. 353). According to their review, these measures assessed the mentor relationship, professional development, characteristics of good mentors, and mentee outcomes in survey-mode. Mentee outcomes were measured using categories such as published papers and securing grant support along with faculty appointments. Meagher et al., highlighted that few qualitative measures of mentor outcomes were discussed in research. This observation makes our study timely and valuable; qualitative data could offer insights regarding the core of mentoring experiences. However, quantitative evaluation, which measures outcomes as a function of multiple individual and environmental factors, have historically been considered important determinants of success.

Johnson (2003) proposed a triangular model of mentor competence as a framework for understanding competences needed for the mentor role in academia. His model included mentor characteristics and intellectual/emotional abilities, as well as the knowledge and skills (competencies) obtained through experience. Nick et. al (2012) proffered a model of excellence in academic mentoring. This model, comprised of six themes to assist faculty members in becoming effective mentors such as (a) identifying appropriately matched dyads, (b) providing clear purposes and goals at the onset of the mentor relationship, (c) solidifying the dyad relationship, (d) providing advocacy and guidance for mentee, (e) integrating the mentee into the academic culture, and (f) mobilizing institutional resources for mentoring support. For the purpose of our study the following definition for mentorship was adopted: "a one on one reciprocal relationship between a more experienced and knowledgeable faculty member (the mentor) and a less experienced one (the protégé) that is characterized by regular/consistent interaction over a period of time to facilitate protégé development" (Haggard, Dougherty, Turban, \& Wilbanks, 2010).

Several researchers have reported that mentoring can be both effective and detrimental (Eby \& Allen, 2002; Eby, McManus, Simon, \& Russell, 2000; Eller, Lev, and Feurer, 2014; Straus, Johnson, Marquez, \& Feldman, 2013). Straus, Johnson, Marquez and Feldman (2013) explored the mentor-mentee relationship, while trying to identify the characteristics of effective relationships and factors that promoted successful interactions. Effective mentoring relationships were characterized by reciprocity, mutual respect, clear 
expectations, personal connections, and shared values. Eller, Lev, and Feurer (2014) suggested that confidentiality, trust, understanding and positive expectations were also vital to effective mentoring relationships. Researchers cautioned that differences related to diversity and communication problems could impede the working relationship and, thus, must be recognized and addressed (Bickel \& Rosenthal, 2011). However, Kim (2014) argued that a good match between mentor and mentee was effective only when the developmental networking is established.

Eby and Allen (2002) developed a taxonomy of the negative experiences that protégés received during mentoring. Those included five domains (a) match within the dyad; (b) distancing behavior; (c) manipulative behavior; (d) lack of mentor expertise; and (e) general dysfunctionality. In another study, Eby and Allen (2002) described negative mentoring experiences encountered by 242 protégés. Those experiences clustered into two factors: distancing/manipulative behavior and poor dyadic fit. Negative experiences were related to job satisfaction, turnover intentions and stress for the mentees. Sometimes negative experiences emerged from misunderstandings rooted in cultural differences. Straus, Johnson, Marquez, and Feldman (2013) suggested that negative relationships included poor communication, deficiency of commitment, personality differences, perceived (or real) counteraction, conflicts of interest, and the mentor's lack of working experience. In sum, the research on evaluation of mentors has shown that (a) there are varied models for evaluation, (b) measures for mentor input and mentee outcomes exist, (c) mentoring can be detrimental as well as beneficial for both sides, and (d) cultural differences need to be taken into consideration when evaluating the efficiency of a mentoring relationship. The purpose of our study was to describe mentees' and mentors' perspectives of how team science promoted collaboration and the development of independent researchers.

\section{Methods}

\section{Research Approach}

A qualitative approach was selected for this study because of its potential to develop insight and deeper meanings about participant experiences relative to team science and mentoring. Authors used inductive analysis to explore mentees' and mentors' perspectives of how team science endorsed collaboration and the growth of independent researchers. Inductive analysis involves, a search for themes that emerge as being important to the description of the phenomenon. It involves several steps including (a) coding and seeing particular text as an important prior to the interpretation, (b) organizing the data to identify and develop themes, and (c) thematic identification, a process that emerges from pattern recognition within the data. Inductive analysis is the process of identifying the patterns, themes, and categories that emerge out of the data rather than being imposed on them prior to data collection and analysis. The method is driven by what the researchers want to know and in the context of this study was guided by the interview questions and participants' subjective descriptions of their experiences (Saldana, 2013). 


\section{Participants}

The researchers used an institutional database of $\mathrm{T} 32$ program information at a researchintensive university in the southeastern United States that listed the names and emails of nine faculty T32 mentors and eight postdoctoral T32 scholars. The researchers invited these individuals via email to participate in semi-structured interviews. Of those who were invited, a convenience sample of five mentors $(55.6 \%)$ and five post-doctoral scholars $(62.5 \%)$ responded to the invitation and agreed to participate in our study. The mentors included two males and three females, all White. All T32 post-doctoral scholars ages 23 and older, were White, and included three males and two females

The process of getting hired into a mentor's laboratory follows. The set of funded T32s grants across the academic health science center requires a list of mentors with known interests Mentees who were interested in working with a specific faculty mentor initiated contact with that mentor. To formalize the mentoring relationship, the mentor's department to which the T32 scholar was assigned had to stipulate and acknowledge that T32 fellowships were awarded to aid the scholar in the pursuit of her research study and that the recipient was not expected to perform services as a condition of this award.

Each T32 program awarded to the academic health science center usually has a recruitment plan or committee. A variety of methods are used to link mentors with mentees ranging from website announcements to emails and communication with associated disciplinary organizations. Postdoctoral trainers usually interview in a laboratory with a space, equipment and funds that can accommodate the postdoctoral fellow's research interest. There is often a standardized application form for each program. The mentor and a committee usually interview all interested postdoctoral fellows to assess their suitably, experience and fit with the potential mentors. Also, it is advised that all fellows have created an independent plan of development (IDP) by the end of the first six months and that is developed in conjunction with the mentor. The IDP specifies what accomplishments the mentor will achieve and how the mentor will assist. In this way, it serves as a guide to evaluate the mentee's progress usually twice a year, as a mechanism to assess the mentee's and mentor's pre-established goals.

After being hired by a mentor, the mentee was paid a biweekly salary and received an opportunity to pursue her own research interest. Mentees met with mentors at intervals determined by the mentor. Mentees developed a committee to guide their research and development. Those committees included the primary mentor to whom they were assigned and one or more secondary, co-mentors, with complementary expertise. Mentee committees met annually or every six months to review the mentee's progress towards attainment of research goals. Engagement with a mentor typically required participation in weekly group meetings and journal clubs to review published studies and other activities as advised by the primary mentor and/or the scholar's committee. In our study, the mentors were basic scientists or clinician faculty. 


\section{Data Collection}

The first author conducted all of the interviews. Prior to our study, the interview questions were reviewed by four health science center faculty all of whom were principal investigators on their own grants and have worked with doctoral and postdoctoral scholars for more than two decades. Interviews ranged from 17-55 minutes. After receiving informed consent (IRB\# 2015-U-0988), the interviews were conducted and recorded online using Zoom technology. Prior to beginning the interview, the lead author re-stated the purpose of the study. During the interview, mentees were asked (a) to describe the benefits of participation in the T32 program, (b) to explain how the didactic/classroom information and laboratory experiences informed their research, (c) to describe how their mentor assisted them in developing their research studies, (d) if they thought that the T32 program would help them obtain grant funding, improve their proposal writing or avert scientific misconduct, and (e) to describe how their level of collaboration changed as the result of participating in the T32 program, as well as how their application of scientific knowledge increased and how their application of translating scientific knowledge changed. During interviews, mentor were asked to (a) describe their goals for mentoring T32 scholars, (b) describe previous mentoring experience and to identify qualities of an effective mentor, (c) state expectations of their T32 scholars, (d) indicate how often they met with the T32 scholar, (e) describe a particular set of skills/abilities that they believed were essential to acculturating T32 scholars into translational science and state why, (f) explain how they planned to facilitate the T32 scholar's ability to acquire those skills/abilities, and (g) explain how that the experiences they provided in formulating questions, designing investigations, laboratory experience, working with data, technologies, protocols or conducting actual laboratory experiences, constructing explanations, testing explanations against current scientific knowledge, and communicating results such as presenting them to others, at a conference, assisted in the development of T32 scholars as independent research scientists. A doctoral candidate in counselor education who was unassociated with the data collection and unaffiliated with biomedical sciences, but experienced in qualitative analysis transcribed the interviews verbatim.

\section{Researchers' Perspectives}

The research team for our study included one faculty member (an experienced qualitative and published researcher from the College of Education) and one doctoral candidate in counselor education. The first author is an experienced qualitative and educational researcher who studies outcomes that accrue from pedagogical interventions, and explores changes in faculty beliefs and teaching, educational research, and assessment practices. Her research initiatives encompass faculty development, cultural competency, and the assessment of behavioral, cognitive, and attitudinal change. The second author is a doctoral candidate in counselor education who is a research assistant for the first author whose has training and expertise in social psychology. The researchers' interest in this study emanated from their observation of inconclusive findings reported in the literature relative to the effectiveness of mentoring and a lack of in-depth qualitative studies about mentor and postdoctoral mentee relationships in team science. 


\section{Data Analysis}

Prior to the analysis, pseudonyms were assigned to each participant. Both the first author and the doctoral candidate read each of the mentee and mentor transcripts line by line as a separate entity and formulated a sense of emergent themes. Next, they met to discuss their independent notions of the emergent themes for the set of mentee and for the set of mentor transcripts. They developed a preliminary and mutually agreed upon codebook for the set of mentee and mentor transcripts. Conceptual definitions were developed for each theme.

Independently the researchers used the codebook to tag text that linked excerpts related to each theme for each transcript (Thornberg \& Charmaz, 2014; Saldana, 2013) The researchers also reviewed the tagged text to ensure their agreement with the themes. The use of two independent coders aided in reducing the potential for interview bias. Reading lineby-line and coding segments assisted in making supporting quotations more accessible. Data were analyzed inductively by coding and identifying participants' spoken words to support the categories that were identified. The methodical process of coding supported by direct quotes guided the researchers' decision making. This rigorous and systematic approach allowed the researchers to feel confident that what they report is representative of participants' perspectives (Hatch, 2002).

Efforts to establish trustworthiness and reduce interview bias were aided through attention to credibility, transferability and confirmability. Credibility, or confidence in truth of the findings, was established through triangulation and peer debriefing. Triangulation was accomplished by using (a) two analysts, (b) 10 interviews, and (c) qualitative analytical tools including line by line coding and peer debriefing to ensure the accuracy of interpretations. Transferability, or the degree to which results of qualitative research can be transferred to other contexts or setting, was addressed by using the same cohort group in the analysis of mentee and then mentor interviews across participant groups (Lincoln \& Guba, 1995). Confirmability was achieved by engaging more than one person in analyzing the data. Validation of the analysis was enhanced by the presence of two experienced qualitative researchers with extensive knowledge in qualitative research (Creswell, 2015).

\section{Results}

Three themes emerged for the mentees (a) working with others, (b) evaluating mentoring and (c) exploring resources. Five themes emerged for the mentors: (a) expanding scholarly productivity, (b) mentoring style, (c) setting team culture, (d) strategies to develop T32 scholars, and (e)evaluating program qualities (see Table 1). The three themes that that depict the mentees' experiences of mentoring and team science are first described. Next, the five themes that characterize the mentors' expectations and approaches to training are described.

\section{Mentees' Experiences of Mentoring and Team Science}

Working with others.-The mentees' experiences in team science were strongly influenced by the nature of collaborations they shared with faculty mentors located inside and outside their department. Mentors frequently encouraged postdoctoral scholars to collaborate with faculty outside the mentor's department to improve the quality of the mentee's proposed research study and to enhance their development as an independent 
research scientist. For example, Thomas explained that while collaborating with others outside of medicine, he developed the capacity to approach problems that exceeded his reliance on a singular point of view. He began to capitalize on "the collective viewpoints and take a more comprehensive approach to considering how the design of his own study could be improved." Having had interactions with faculty outside his assigned department in medicine broadened his thinking. He expressed his appreciation for learning how research questions could be explored using "completely different perspective[s]." In previous training, Thomas worked primarily alone. In that environment, he found that a lack of resources and physician's inability to engage in research essentially quashed the potential for collaborative research. Within the team science framework, participants received abundant opportunities to network with other faculty in and outside their assigned department and to interact with other postdoctoral and graduate students in their mentor's research group. These experiences fostered (a) formal and informal discussions about the T32 scholar's research goals, (b) sharing of formative successes and failure they faced along the way and (c) opportunities to address scientific questions. Owing to team science and a dedication to multidisciplinary interactions, one of the prominent training activities offered in this program, Thomas came to cherish how involving other people provided new perspectives and ideas about viewing and approaching research problems. Concurring, Earl valued the open communication between clinicians and basic scientists, an activity in which his mentor urged him to partake. Participants reported how consultations with colleagues and interactions with others during journal club and seminar meetings forced them out of their comfort zone. Earl proffered that listening to others' ideas caused him to have more questions and required "me to do a lot more reading on things I never did before."

Thomas explained that the program accorded a mechanism to incorporate concepts outside of clinical medicine and encouraged him to begin thinking about how to relate his research project to other public health areas. Taking patient-centered research to a more global level and pondering its application to a larger scale was in his estimation, one of the benefits of multidisciplinary collaborations. Specifically, he deliberated about how he could utilize bioinformatics or the repository to work with different translational science researchers in conjunction with his research model.

Diane heralded the T32 training for broadening her horizons. At her previous institution, her research, which centered on identifying alcohol induced brain damage and related behavioral outcomes and emphasized the development of interventions, would have come to an end. Prior to the T32, she did not know how to apply her research ideas or outcomes, or seek funding. She described the instrumentality of her mentoring and training. Diane received guidance that enhanced her understanding of funding and how to write grant applications. Also, her mentor helped her recognize the type of statistical knowledge she needed to ensure that she had "a background of funding, publications and a good study underway." Having had a multitude of team science experiences such as opportunities to seek input from other faculty, she felt confident of her readiness to move into a faculty position. She also described how interactions with patients "stroked my passion for the research" in contrast to those with other individuals or with textbook learning. Her mentor ensured that Diane acquired requisite new knowledge and clinical experiences that allowed her to attain her research goals. Diane surmised that as a result of engaging with faculty across several disciplines 
whose expertise aided her research interest in addition to the mentoring provided by her primary and secondary mentors, that she could now operationalize many of the ideas that previously had "float[ed] around in my head."

Not all mentees enjoyed universally positive interactions with their mentors. Beverly sought the training grant to augment her laboratory knowledge on hypertension and to acquire a knowledge base regarding obesity models. Shortly after starting her training, her primary mentor retired from the university. His retirement effectively forced her to begin working independently. As a post-doctoral fellow, she worked with four young junior faculty members who were all principal investigators (PIs) on their own grants. She described the advantages and the limitations associated with her unique circumstance. As a collective of five individuals they were able to share a plethora of information at meetings, and at this level provided a form of mentoring. However, she asserted that this process was overwhelming and that often, it was hard to tailor meeting discussions to any one specific research study. Elaborating, Beverly proffered that competition among faculty made decision-making problematic, because each person ended up advocating for his/her own research agenda and thus, were unavailable to assist Beverly with advancing her own research. Beverly's mentor was off campus and unavailable on site on a day-to -day basis. Yet, other faculty in her research group could have provided some type of intellectual assistance. However, as seen by Beverly's description, they did not. Given the perspective that the mentor-mentee relationship is typically directed towards helping the less experienced colleague move towards a desired level of competency, it can be seen how these circumstances were particularly challenging in Beverly. This circumstance was further compounded by the absence of Beverly's primary mentor, who had retired. Thus, she was unable to seek the counselor of the primary mentor in a timely manner.

Another mentee, Robbie, did not hold his mentoring experience in high esteem. He explained that his primary mentor in medicine and his secondary mentor in molecular genetics molecular biology did not communicate about his research goals. Robbie opined that, "maybe one of them assumed that the other person was taking care of the training aspect and stuff relevant to the T32." He implied that a lack of communication and assumptions led to his unsatisfying experience. However, Robbie also suggested that competition in the field played a role He shared that, "from what I've understood only $10 \%$ of postdocs who come in actually get a [position] in academia [or] become a principal investigator." There was also a lack of understanding related to what Robbie wanted to do and what his primary mentor thought he should be doing. His primary mentor believed that his computational background and expertise was his real strength. His mentor stated that Robbie's "strengths [are] in one area and he really wants to do something else.[He is ]really good in computers, programming and IT and yet whenever I try to give [him] more work and informatics and data analysis [he doesn't] want to do." Robbie, on the other hand, wanted to engage in laboratory research. The mentor and mentee did not hold similar expectations for his training.

Evaluation of mentoring.-Mentees' descriptions of mentoring ranged from highly satisfied to considerable disappointment. Diane explained how her mentor's outreach to another colleague added expertise to her study. Her mentor initiated contact with a colleague 
who had expertise in neuroimaging and was using a social cognitive perspective in his research, another example of how team science facilitated postdoctoral mentees research interests. In addition to her mentor's background, this colleague added expertise in areas that Diane did not have.

Diane conveyed that she entered into the T32 program with a solid foundation in research and many publications. However, she lacked familiarity with the addictions literature. With guidance and direction from her mentor, she became conversant with this body of work and with relevant neurological structures. New knowledge was pivotal in informing her curiosity regarding how damage secondary to alcohol altered problem recognition. For example, while she knew the brain regions that she wanted to explore, she did not have requisite knowledge of what regions supported the brain concept. Because of her training, intensive study and mentor guidance, Diane's "scientific knowledge has grown exponentially."

Thomas was fortunate to have a primary mentor that helped to ensure that he was surrounded with other team science investigators who were devoted to understanding the processes related to research design, research development and collecting the data. $\mathrm{He}$ asserted that as a result of his mentoring he acquired "a new level of respect for research." Using this new information from many interactions with his primary mentor as well as other scientists across disciplines, Thomas revised his research design to permit a generalizable approach in the hope that his findings would impact more people than simply "people in my own clinical setting." Aside from developing more robust research designs, the experiences that his mentor directly provided or those that his mentor encouraged him to seek, sharpened his research focus and abetted his development of more efficient research plans.

In part, owing to her mentor's retirement, Beverly's mentoring experience was not optimal. Her research interests were not centrally located in one college department and in fact cut across two departments in two colleges. This presented unforeseen obstacles and proved to be divisive. Her research expertise in hypertension was located in the medical school's physiology department. However, she was assigned to the department of pharmacology in the school of pharmacy. Because of differences in faculty pursuits and cultures, faculty in these departments did not meet or work together. A lack of cross-departmental interactions with her mentors contributed to her sense of isolation. She had to take the initiative to locate faculty whose research interest contributed to her interest and find faculty with whom she could discuss her science. Nonetheless, Beverly was grateful for her lab experiences. By becoming a T32 scholar, she attained the knowledge of obesity that she sought and needed. In retrospect, she wished that she had been paired with a mentor whose career interests were not so established. The work of Beverly's initial mentor was primarily in obesity, a major risk factor for cardiovascular disease. When she tried to apply her cardiovascular experience with hypertension to her mentor's knowledge of obesity, he had no idea on what she was doing. His lack of cardiovascular experience seriously hampered efforts to integrate her expertise in hypertension with obesity.

Robbie offered little positive commentary regarding the mentoring he received and there was little commentary to suggest that he received a satisfactory team science experience. $\mathrm{He}$ likened the T32 training to an insurance policy that ensured that he had a job. Moreover, he 
explained that the funding had little to do with the continuity of his research. He admitted that his research could have continued "with or without the T32." From his interview, it seemed evident that he had not benefitted from this experience in any tangible manner. Rather than acquiring new knowledge or skills, he proclaimed that the new skills he developed actually resulted from his postdoctoral studies and his independent research, not from the T32 experience per se.

Exploring resources.-Interactions with other faculty were catalysts in getting participants to think more broadly about the application of their research. Earl described how questions from other faculty challenged cogitating about his research project. Once while describing how the nerve worked, one of the faculty retorted, "I don't care how the nerve works, I want to know if this will change things for the clinical population." This question prompted Earl to reflect differently about applying his basic science knowledge and to wonder how he could extend the outcomes of his research to clinical care. Prior to the T32, Earl worked alongside his mentor in the same office in a small room. Unlike his previous experience, affordances to networking opportunities were a staple in the T32 training program. While commenting on his own expertise, Earl proffered that he had raised his mentor's ability to explore clinical research by helping him "look at more rigorous science questions."

The findings showed how the T32 related-resources benefitted the mentoring experiences that postdoctoral mentees received. Resources included specialized training seminars, attending conferences, access to patients, and the dedicated use of particular equipment or methodology. Earl explained how the K College helped scholars develop grant writing skills. He also acknowledged how opportunities to discuss grant proposals with both of his mentors aided his growth in eventually writing his own grant.

Mentors encouraged mentees to attend meetings in their research specialty, to become acquainted with what leaders in their specialty area were studying. Beverly referenced the benefits afforded by attending national meetings. She asserted that presenting her data and interacting with top people in the field of hypertension really "helped me to tailor my data." Attending the conference afforded her additional insight, that otherwise she might not have received. Having her mentor suggest attending the conference and discussing her findings with others, was one benefit of having a designated mentor. Moreover, she became aware of resources that she did not previously know about. This opportunity was paramount to the development of her research project. Colleagues advised her to do telemetry. After relating that the university's devices were out of date, they offered to let her use their equipment. She elaborated about how she benefitted from collaboration when others gave her advice about establishing "hypertension in an obese model." Beverly also described the new surgical techniques that she had learned such as braising lamping injections. However, she expressed disappointment after discovering that lab she joined did not have the basic gene therapy she thought would be available.

Earl was thrilled about the availability of particular equipment. Through this experience he was able to explore pain modulation in unique ways. He was able to observe certain nerve activations or how particular stimuli evoked the system differently using the same pathways. 
Expounding, he shared that access to these specialized resources allowed the group "to answer things that no one else in the world can do." His mentor's laboratory took baseline and dynamic measures over time that were moderated by exercise. As Earl pointed out, this approach provided a more a clear picture of a pain as a disease and its influence on nerves. Another aspect of his training was expeditious access to publications. He reported that his primary and secondary mentor had already amassed over 300 papers.

\section{Mentors' Expectations and Approaches to Training}

Mentors described their approaches to training independent researchers through expanding scholarly productivity, mentoring style, and the process of setting goals for a team. They also described the strategies they used to encourage the development of T32 scholars and to evaluate programmatic qualities.

Expanding scholarly productivity.-Fred expected his scholars to show a certain level of scholarly productivity, dependent on the kind of research they were doing. Submitting multiple manuscripts per year as well as an application for their own independent funding within the first year and a half was another requirement he established. Over time, to ensure their potential for productivity, he insisted that scholars take full advantage of training opportunities inside and outside his laboratory to get the most from their experiences. Like Fred, Jacqueline expected T32 scholars to write three to four papers a year, although not as first author. She also required them to submit a grant and help others write grants. Sandra's aim was to aid trainees in the identification and achievement of their personal career goals. Ultimately, she hoped that her T32 scholars would be motivated to seek academic research careers or commit to engaging in ongoing research.

Mentoring styles.-Max was very directive in his approach to mentoring. He set strict deadlines for manuscript submissions. He parsed out the writing tasks for sections within the paper and expected scholars to deliver written work in concert with pre-established goals. Max held scholars accountable for follow through. Using a different approach to writing, Jacqueline shared her belief that shadowing was essential to teaching. Amplifying this point, she explained that when her group wrote grants, they projected written sections on the screen and then they would sit down and complete the task together.

Setting team culture.-While cultivating an appreciation for team science, Nina, fostered a belief about the importance of teamwork by helping scholars realize how their work fit into the goals of the overall research project. To her, collaboration was an essential life lesson. Sandra called attention to the necessity of sharing the workload. She accentuated that research outcomes did not rest solely on the efforts of one person. She believed that everyone needed to appreciate "that science is team science." Exemplifying this belief, she ensured that her postdoctoral scholars were cross-trained to work in treatment facilities and knew how to conduct group screening. Training warranted that scholars develop appropriate interpersonal skills to communicate with patients, that they understood the purpose of the behavioral studies and recognized why the research team asked particular questions. Jacqueline's perspective centered more on the role of communication during interactions. She pointed out the necessity of being kind and thoughtful to everyone on the team by 
stating that it was important to be as "nice to the janitor as you are to your mentor" because "everybody is important on the team."

Strategies to develop T32 scholars.-Mentors described the ways in which they encouraged the development of scholars' capacity for scientific thinking. Sandra described circumstances when scholars proffered hypotheses that were unsupported by preliminary data. This was one of many times when she challenged their reasoning skills. She encouraged students to develop thinking skills such as identifying the implications in instances when an interpretation was right or wrong. In this way, she strived to ensure that they could offer evidence-based support for their reasoning. Sandra surmised that if scholars were unable to engage in this type of thinking, then translational science work was not likely possible. Nina suggested that scholars cultivate a willingness to abandon prevailing assumptions and biases. She urged that they familiarize themselves with the current literature. Nina also asserted that procuring an understanding of the scope of the field was absolutely fundamental to maturation in thinking.

Jacqueline endeavored to provide scholars with hands on experiences that would help them crystallize and move towards career goals. By providing this type of learning environment, she hoped to offer the type of context that would motivate scholars to develop independent research careers. Fred used teaching instances to query scholars about their research questions. He challenged them to articulate the overall goal of their research and to describe the underlying hypothesis that they planned to test. He also expected them to elaborate upon the ways in which they could design their study. Fred also used discussions of research publications as opportunities to engage scholars in critique. He asked scholars to identify the research questions and to explain how the study was designed to answer those questions. Jacqueline stressed that research collaboration begin early and suggested nurturing those collaborations so that they would sustain throughout one's career. She offered examples of writing with the same colleagues for more than 40 years.

Max encouraged his postdocs and students to collaborate with other faculty and other scientists "as much as they can," emphasizing his belief that it's a "very important part of their development." He also tried to ensure that his postdoctoral scholars received and provided mentoring to others including junior, graduate, or master's level students. In terms of providing resources, Max paid for one of his students to travel to a lab at an off-campus site for two weeks, to acquire techniques, bring them back to the lab, and teach them to everyone else.

Often Fred would give scholars a data set matching their interest and then encourage them to get help analyzing the data. Invariably, as he pointed out, it was feedback that scholars received during the analysis that helped them isolate the gaps in their knowledge, research or statistical skills, or the missteps in how they were stating their research questions. Max also concurred with the merit of faculty-scholars' interactions, proclaiming that it caused scholars to explain their model, procedures and results clearly and simultaneously defend their reasoning. Nina, agreed and suggested that subjecting scholars to the "wisdom of crowds" and their unplanned critique offered "really good teaching circumstances." 
Evaluating program qualities.-Several mentors pointed out concerns about the limitations of training opportunities or the fit of the postdoctoral fellow with the program. Nina opined that the program needed to bolster the quality of its statistical training. She asserted that it was "not as good as it should be." She believed that additional training in molecular biology was necessary along with content on business principles so that scholars would learn about budgeting and living by a budget. In contrast, Nina appreciated the program's recent emphasis on "training our T32s in non-traditional areas." Fred mentioned the importance of recognizing whether or not the prospective T32 scholar was really a fit for the program. He pointed out that there needed to "be an interest and commitment," opining that this was not something that "you can teach."

\section{Discussion}

We provided insight about how mentoring experiences and engagement in team science fostered the development of independent researchers. Almost allof the participants agreed that the T32 program provided a conduit for accessing support for their research and for providing an environment to do that work. Faculty mentors in the T32 grant are "practicing what they preach" as Wildman and Bedwell (2013, p. 381) advocated. T32 scholars were engaged in an atmosphere of collaboration fostered by their mentors who constantly monitored their development and provided sufficient challenge and support to raise their capacity for independent investigation.

Findings of the current research addressed all three levels of analysis suggested by Fiore (2012). At the organizational level, the T32 program provided scholars with transparent boundaries with regards to reaching out to collaborators from outside the health sciences and even outside of the university to help them capitalize on the collective viewpoints of research design, which aids the multi-disciplinarily focus of the T32 program. At the level of the task, mentors and mentees were constantly involved in identifying the methodology or type of data analysis used in publications they reviewed through regular meetings and journal clubs. Most participants acknowledged the need for optimal communication and solid organizational planning so that the research team continued to thrive. This finding, aligned with previous research, highlights the necessity for explicitly communicating expectations for the mentees on team participation, along with clarifying expectations of the mentees (Bennett, Maraia, \& Gadlin, 2014). At the team analysis level, four of the five mentors sought training opportunities for the mentees mostly within and some outside the program. This process seemed to be mutually beneficial to mentees and mentors. Sometimes mentees asked to be sent to another program or university to learn a new technique. Yet in so doing, they were expected to bring it back to the team so that it enhanced its productivity.

Both mentors and mentees described positive mentoring experiences when both sides held similar expectations with regards to satisfying mutual needs in the research process. When the mentors and mentees expectations of one another and of the relationship matched, team functioning seemed to improve. Mentees articulated their interest in and appreciation of learning from mentors and in acquiring access to scientific and educational resources that was sufficient for their research and career development. Mentors, in turn, were motivated in moving their research team forward and in cultivating independent investigators. The 
findings suggest that overall, mentors provided advocacy and guidance for mentees, integrated them into the academic culture, and mobilized institutional resources for mentoring support. The findings also showed how multiple mentorship may be key for the T32's scholarly development confirming the findings of de Janasz and Sullivan (2004) who argued that the one-mentor model is not realistic, as the portfolio of mentors facilitates a variety of experiences. This suggestion is supported by the realization that an emphasis on multidisciplinary translational science is a relatively "new concept to many academic departments and often requires identification of mentors outside the home" department (Hacker, Subramanian, \& Schnapp, 2013, p. 414). Moreover, as seen in two of the mentees' experiences, reliance on a model of single mentorship can be detrimental when a mentor and a mentee cannot find mutual ground in collaboration, goal settings or the frequency of interactions.

Having only one mentor can result in mentees becoming trapped if they have communication problems with this person, which was manifested in interactions and perceptions of one another's goals and abilities. Having two mentors, which is a common characteristic in team science may be helpful for the mentors themselves as Dziczkowski (2013) argued. Personal and societal constraints have demonstrated that mentoring takes extensive time, effort, and commitment. Therefore, having a helping hand from another mentor may be beneficial. This concern was aptly illuminated by two mentees who did not benefit from their collaboration with mentors. Those experiences might have been obviated had they been assigned to more than one mentor. Concurring with what has been reported by others (Eby \& Allen, 2002; Eller, Lev, \& Feurer, 2014; Straus, Johnson, Marquez, \& Feldman, 2013), such as in the case of Robbie, the mentee's and mentor's conceptualization of the mentee's abilities was distinctly different. This dynamic led to a lack of communication, confidentiality, and trust, and possibly hidden conflict with mentee who suppressed his dissatisfaction due to his lower place in the social hierarchy (Eller, Lev, \& Feurer, 2014). This finding also aligned with Kim (2014) who reported that a developmental network is necessary to the match between a mentor and mentee. The absence of this framework can result in dissatisfaction when a mentor and a mentee see different paths for the mentees growth and developmental goals for the mentee differ. In case of Beverly, her mentor was on site for only about the first month after she began working in his laboratory before he retired. Despite being surrounded by several young faculty in the laboratory, her relationship with them was often strained by a culture of competition. Participants, both mentees and mentors, also offered several suggestions to strengthen training experiences that would enhance their development as independent translational research scientists.

\section{Recommendations and Implications of the T32 Program}

Both mentees and mentors offered insight into the ways that the T32 program could be improved. Mentees, for example, recommended assessing the scholarly activities of those faculty who were mentors and were also at the end of their career. They suggested making a careful determination regarding whether or not mentor who planned to retire while the T32 was in the program should take on new postdoctoral scholar. They pointed out the necessity of discerning whether or not end of career faculty had the ability to support the mentee's intellectual development and to consider whether or not they were working on novel things. 
Along the same lines, Fred recommended determining the fit between prospective postdoctoral scholars and the mentor's group they planned to join One inference that can be drawn from the findings is that fit between mentor and mentee was dependent on several interrelated factors (a) the mentor's expertise, (b) ensuring that the mentee's previous experiences, interest and potential for becoming an independent researcher was matched with the program and the assigned primary mentor and (c) that mentor and mentee held similar expectations for the mentee's growth and productivity.

Perhaps developing a set of criteria for selecting applicants and placing them with particular mentors would be helpful in this regard. Towards this aim, the researchers suggest a review of the application process for accepting a post-doctoral T32 scholar and ensuring the appropriateness of placement with particular mentors. Components of the application process should focus on the mentee's (a) track record, (b) academic record, (c) feasibility of the written research plan and timeline, and (d) significance of the research plan including its feasibility and the potential of translation of the research project to humans, patients, practice and community health needs. Criteria for the mentor should include (a) expertise of the mentor, (b) track record of prior mentees, (c) current research funding, (d) evidence that the mentor is familiar with the applicant's proposal and research plan and has intellectual and financial expertise to support it and (e) evidence of research funding. Adopting this type of application and review processes would help ensure the fit of the scholar to particular mentors and transparency of the process.

Several mentees discovered that certain laboratory or research-related resources were not available. Others were disappointed to find certain resources, which they assumed were available, did not exist, or were outdated. These finding suggests the important of being thorough and transparent during communications with potential postdoctoral scholars regarding the academic, clinical and laboratory resources before asking them to sign a letter of appointment. Developing a description of each laboratory, the mentor's research agenda, the type of work supported by the laboratory, and the specialized equipment housed would aid postdoctoral scholars in deciding whether their research interests were matched.

\section{Limitations of the Study}

Our study findings are limited to those mentees and mentors who elected to participate in the interviews and the degree to which they were willing to disclose their experiences. Also, only one interview per participant was conducted so the interpretations are limited to the available data. However, in contrast, a total of 10 interviews were conducted. Also, two experienced qualitative researched coded the data were independently.

Hall et al., 2008 reminded that [the success of the research team] "depends on the needs of all the stakeholders including investigators, post-doctoral and graduate students, and research support staff" (p. S246). Individuals are likely to feel intimidated and reluctant to share the truth about the teamwork with the researcher if they feel that disclosure might influence their position on the team. Feedback generally changes group dynamics and it is common knowledge, that conflict can imperil development moving forward. Therefore, at the organizational level, it is essential to develop a culture that encourages team science 
participants to feel confident that authentic feedback, both positive and negative, would be welcomed and would not have a deleterious impact.

Future studies should focus on discovering the perceptions of each team science member, including the primary mentor, other postdoctoral, graduate, and undergraduate students, laboratory technicians, and administrative staff, to explore how the team functions as a group. Skilled qualitative researchers are poised to assure team members opportunities to share candidly without fear of disclosure. Exploring what and how group dynamics aids the overall success of the team and what factors impact the ability of T32 scholars to become productive and successful team players would be an important contribution to understanding team science and the role of effective mentoring. Future studies that explore how mentoring impacts researcher productivity and pathways towards the development of independent research scientists in team science should (a) explore how mentees establish proper communication with their mentors, (b) investigate the phenomenology among the mentees as they seek to find a balance between multiple mentors, and (c) study how mentors help mentees embrace the pathway towards an independent career. Notably, mentors in this study mentioned independence more frequently than mentees. For Beverly, independence had a negative connotation, as she felt abandoned by her mentor. Therefore, advancing an understanding of how mentor-mentee interactions and experiences foster researcher autonomy might better illuminate the scholars' development towards independence.

\section{Conclusion}

Federally-funded training programs, such as the NRSA, are obliged to demonstrate effectiveness in preparing postdoctoral scholars achieve independence as translational scholars. To a large extent, the success of this and similar programs is broadly dependent on the primary and other mentors to whom the scholar is assigned (Fleming, Burnham, \& Huskins, 2012). Understanding the quality of the mentoring relationship, the types of communication styles and nature of interactions is central to assessing program effectiveness. Based on the findings, it seems unclear that there was a formalized process for aligning expectations between mentees and mentors such as Individual development plans or formal agreements. Neither the mentees or mentors made reference to such types of agreements for setting up mutually agreed upon goals. In light of this finding, it is recommended that all T32 programs initiate a practice that formalizes agreed upon mentormentee expectations and that this compact be revisited by an external committee to assess the T32 scholar's progress. Validated measures of the mentor's satisfaction with the mentees' progress and the mentees' satisfaction with the mentoring received should also be completed annually. The institution should also implement competency-based evaluation of research mentors using validated measures and assessment procedures (Meagher et al., 2011). Perhaps all mentors engaged in training the next generation of clinical translational researchers should be required to complete mentor training before being assigned a postdoctoral scholar or they should be required to participate in some form of mentor workshops, seminars or training while advising T32 scholars (Feldman, Huang, \& Guglielmo, 2009). Overall, we highlighted an important role for qualitative research. The findings demonstrated outcomes related to investments in large science groups (e.g., research centers) enhanced our understanding of how best to support and manage team 
science, and illuminated how team science mentoring relationships may promote collaboration and the development of independent researchers.

\section{References}

Bennett LM, \& Gadlin H (2012). Collaboration and Team Science: From Theory to Practice. Journal of Investigative Medicine: The Official Publication of the American Federation for Clinical Research, 60(5), 768-775. http://doi.org/10.231/JIM.0b013e318250871d [PubMed: 22525233]

Bennett L, Maraia R, \& Gadlin H (2014). The "welcome letter": A useful tool for laboratories and teams. Journal of Translational Medicine and Epidemiology, 2, 1035-1045 [PubMed: 28649581]

Bickel J, \& Rosenthal SL (2011). Difficult issues in mentoring: recommendations on making the "undiscussable" discussable. Academic Medicine, 86, 1229-1234.10.1097/ACM. 0b013e31822c0df7 [PubMed: 21869662]

Borner K, Contractor N, Falk-Krzesinski HJ, Fiore SM, Hall KL, Keyton J, ... Uzzi B (2010). A multilevel systems perspective for the science of team science. Science Translational Medicine, 2, $49 \mathrm{~cm} 24-49 \mathrm{~cm} 24$. doi: $10.1126 /$ scitranslmed.3001399

Byington CL, Keenan H, Phillips JD, Childs R, Wachs E, Berzins MA, ... Clark EB (2016). A matrix mentoring model that effectively supports clinical and translational scientists and increases inclusion in biomedical research. Academic Medicine, 91, 497-502. doi:10.1097/acm. 0000000000001021 [PubMed: 26650676]

Creswell JW (2015). Educational Research: Planning, Conducting, and Evaluating Quantitative and Qualitative Research. 5th Ed. Pearson

Croker A, Trede F, \& Higgs J (2012). Collaboration: What is it like? - Phenomenological interpretation of the experience of collaborating within rehabilitation teams. Journal of Interprofessional Care, 26, 13-20. doi:10.3109/13561820.2011.623802 [PubMed: 22233363]

Croyle RT (2008). The National Cancer Institute's transdisciplinary centers initiative and the need for building a science of team science. American Journal of Preventive Medicine. 35(2S):S90-S93. [PubMed: 18619408]

Croyle RT (2012). Confessions of a team science funder. Translational Behavioral Medicine. 2(4):531534. [PubMed: 24073153]

de Janasz S \& Sullivan S (2004). Multiple mentoring in academe: Developing the professorial network. Journal of Vocational Behavior, 64, 263-283. doi.org/10.1016/j.jvb.2002.07.001

Dziczkowski J (2013). Mentoring and leadership development. The Educational Forum, 77, 351-360. doi:10.1080/00131725.2013.792896

Eby L, McManus S, Simon S, \& Russell J (2000). The protégé's perspective regarding negative mentoring experiences: the development of a taxonomy. Journal of Educational Behavior, 57, 121. doi.org/10.1006/jvbe.1999.1726

Eby L \& Allen T (2002). Further investigation of protégés' negative mentoring experiences. Group \& Organization Management, 27, 456-479. doi.org/10.1177/1059601102238357

Eller LS, Lev EL, \& Feurer A (2014). Key components of an effective mentoring relationship: A qualitative study. Nurse Education Today, 34, 815-820.10.1016/j.nedt.2013.07.020 [PubMed: 23978778]

Falk-Krzesinski HJ, Contractor N, Fiore SM, Hall KL, Kane C, Keyton J, ... Trochim W (2011). Mapping a research agenda for the science of team science. Research Evaluation, 20, 143-156. doi:10.3152/095820211×12941371876580

Feldman MD, Huang L, Guglielmo BJ, et al. (2009). Training the next generation of research mentors: the University of California, San Francisco, Clinical \& Translational Science Institute mentor development program. Clinical Translational Science 2(3):216-221. [PubMed: 19774102]

Fleming M, Burnham EL, \& Huskins WC (2012). Mentoring translational science investigators. JAMA, 308(19), 1981-1982). [PubMed: 23168821]

Fiore S (2008). Interdisciplinarity as teamwork: how the science of teams can inform team science. Small Group Research, 39, 251-277. doi:10.1177/1046496408317797 
Fiore SM (2012). Networking knowledge creation. Science, 336(6077), 36-37. doi:10.1126/science. 1216839

Fiore SM, \& Wiltshire TJ (2016). Technology as teammate: Examining the role of external cognition in support of team cognitive processes. Frontiers in Psychology, 7, 1-17. doi:10.3389/fpsyg. 2016.01531 [PubMed: 26858668]

Guise JM, Geller S, Regensteiner JG, Raymond N, \& Nagel J (2017). Team mentoring for interdisciplinary team science: lessons from K12 scholars and directors. Academic medicine 92(2): 214-221. doi: 10.1097/ACM.0000000000001330. [PubMed: 27556675]

Hacker BM, Subramanian L, \& Schnapp LM (2013). Strategies for mentor matching: lessons learned. Clinical and Translational Science, 6(5),414-416. doi:10.1111/cts.12050 [PubMed: 24127933]

Haggard DL, Dougherty TW, Turban DB, \& Wilbanks JE (2011). Who is a mentor? A review of evolving definitions and implications for research. Journal of management, 37(1), 280-304.

Hall K, Feng A, Moser R, Stokols D, \& Taylor B (2008). Moving the science of team science forward. American Journal of Preventive Medicine, 35, S243-S249. doi:10.1016/j.amepre.2008.05.007 [PubMed: 18619406]

Hatch JA (2002). Doing qualitative research in education settings. Albany, NY: SUNY Press.

Johnson B (2003). A framework for conceptualizing competence to mentor. Ethics \& Behavior, 13, 127-151. doi.org/10.1207/s15327019eb1302_02

Kauffeld S, \& Lehmann-Willenbrock N (2011). Meetings matter: effects of team meetings on team and organizational success. Small Group Research, 43, 130-158. doi:10.1177/1046496411429599

Kim SE (2014). The mentor-protégé affinity on mentoring outcomes: the mediating effect of developmental networking. International Review of Public Administration, 19, 91-106. doi: 10.1080/12294659.2014.887368

Lotrecchiano GR (2013). A dynamical approach toward understanding mechanisms of team science: Change, kinship, tension, and heritage in a transdisciplinary team. Clinical and Translational Science, 6, 267-278. doi:10.1111/cts.12054 [PubMed: 23919361]

National Cancer Institute. Team science toolkit. Accessed February 5, 2017 from: https:// www.teamsciencetoolkit.cancer.gov/public/WhatisTS.aspx/

Meagher E, Taylor L, Probsfield J, \& Fleming M (2011). Evaluating research mentors working in the area of clinical translational science: A review of the literature. Clinical Translational Science, 4, 353-358. doi: 10.1111/j.1752-8062.2011.00317.x [PubMed: 22029808]

Nick JM, Delahoyde TM, Del Prato D, Mitchell C, Ortiz J, Ottley C, ... \& Siktberg L (2012). Best practices in academic mentoring: a model for excellence. Nursing research and practice, 2012.

Ruth L Kirschstein Institutional National Research Service Award. Accessed December 28, 2016 from: https://researchtraining.nih.gov/programs/training-grants/T32.

Saldaña J (2013). The coding manual for qualitative researchers. Los Angeles: SAGE Publications.

Straus SE, Johnson MO, Marquez C, \& Feldman MD (2013). Characteristics of successful and failed mentoring relationships. Academic Medicine, 88, 82-89. doi:10.1097/acm.0b013e31827647a0 [PubMed: 23165266]

Stokols D Methods and tools for strategic team science; Presented at the Planning Meeting on Interdisciplinary Science Teams; January 11; Washington, DC 2013 Retrieved February 5, 2017 from: https://www.ncbi.nlm.nih.gov/books/NBK310382/

Thornberg R, \& Charmaz K (2014). Grounded theory and theoretical coding In Flick U The SAGE handbook of qualitative data analysis (pp. 153-169). London: SAGE Publications Ltd. doi: $10.4135 / 9781446282243$

Wildman J, \& Bedwell W (2013). Practicing what we preach: teaching teams using validated team science. Small Group Research, 44, 381-394. doi: 10.1177/1046496413486938 


\section{Table 1}

Mentee and Mentee Themes and Conceptual Definitions

\begin{tabular}{|c|c|}
\hline $\begin{array}{l}\text { Mentee Themes and Conceptual } \\
\text { Definitions }\end{array}$ & $\begin{array}{l}\text { Mentor Themes and Conceptual } \\
\text { Definitions }\end{array}$ \\
\hline $\begin{array}{l}\text { Working with others - interacting or collaborating with } \\
\text { other faculty, advisors and mentors and engaging in } \\
\text { meetings }\end{array}$ & $\begin{array}{l}\text { Expanding scholarly productivity - -- identifying expectations for scholars' } \\
\text { accomplishments }\end{array}$ \\
\hline $\begin{array}{l}\text { Evaluating mentoring- assessing the quality of mentoring } \\
\text { received }\end{array}$ & Mentoring style- characterizing interactions with scholars. \\
\hline \multirow{3}{*}{$\begin{array}{l}\text { Exploring resources - describing how program experiences } \\
\text { and funding fostered improvements in knowledge or skills }\end{array}$} & Setting Team Goals - instilling an appreciation for team science \\
\hline & $\begin{array}{l}\text { Strategies to develop T32- explaining ways in which mentors encouraged the } \\
\text { development of scholars' capacity for scientific thinking. }\end{array}$ \\
\hline & $\begin{array}{l}\text { Evaluating program qualities - assessing program effectiveness and fit for } \\
\text { scholars }\end{array}$ \\
\hline
\end{tabular}

